\title{
Simulation Analysis for GIS Internal UHF Partial Discharge Signal Propagation Characteristics
}

\author{
Li Wei \\ Dept.of Electrical Machinery and Equipment \\ Shenyang University of Technology \\ Shenyang,China \\ e-mail:cgsmxcz@163.com \\ Cheng Guangsheng \\ dept. of Electrical Machinery and Equipment \\ Shenyang University of Technology \\ Shenyang,China \\ e-mail:cgsmxcz@163.com
}

\author{
Lin Jiusheng \\ Dept.of Electrical Machinery and Equipment \\ Shenyang University of Technology \\ Shenyang,China \\ e-mail: 906934300@qq.com \\ Yang Haiying \\ Dept. of Applied Economics \\ Shenyang University of Technology \\ Shenyang,China \\ e-mail: 827805614@sina.com
}

\begin{abstract}
This paper researches on the propagation characteristics of partial discharge in internal GIS. Through the external UHF sensor to receive the electromagnetic wave signals emitted from the surface of the insulator metal pouring hole flange and using the finite difference time domain method, by means of XFDTD modeling, simulation and analysis the propagation characteristics of partial discharge signals in GIS cavity. The result shows that the UHF partial discharge signal along the GIS cavity axis direction emitted from the metal flange pouring hole has the maximum electric field component; electric field strength modulus value in the center is greater than the modulus of both sides, and both sides are symmetrical distribution; the direction of the magnetic field is parallel to long edge and the strength of magnetic field modulus value in the shortside is greater than the midline of long side.
\end{abstract}

Keywords-GIS; partial discharge; UHF sensor; finite difference time domain; XFDTD

\section{INTRODUCTION}

In recent years, gas insulated metal enclosed switchgear (GIS) assembly is increasing year by year, year-on-year growth rate has exceeded the open type circuit breaker. External UHF sensor can detect the UHF electromagnetic signal under the state of no power cut, which is emitting from insulator metal flange casting hole, after the internal filtering and removed the noise, the amplified voltage signal will be uploaded to the PC conversion in real time for subsequent diagnostic analysis and Technical personnel can confirm types of power supply and discharge, arrange the maintenance time, work out maintenance plan of safety, greatly reduces the failure of power losses. Propagation characteristics of partial discharge signals of GIS is benefited for the receiving sensor design and sensor to high efficiently receive the PD signal from the inside of GIS.

Finite difference time domain method (FDTD) in the transmission of high frequency electromagnetic field, the radiation and scattering problems and projection is used widely. It is a kind of method will contain time Maxwell curl equation transformed into a set of difference equation and electromagnetic field solution space on the time axis gradually to approach. Finite difference time domain method after more than 20 years of development, with its direct time domain calculation mode, extensive applicability, economy of storage space and computation time, procedures of the characteristics of generality and concise, intuitive, standing out from the traditional finite difference method, is widely used in the study of electromagnetic wave propagation related simulation[1-2].

Based on the literature [3-8],using the method of FDTD, this article simulates and analyses a period of GIS cavity waveguide model. The paper mainly studies Electric field intensity, magnetic field intensity and the intensity in different location of insulator metal flange pouring hole, through the research of the above problem, provides guidance for the installation of the external UHF sensor detecting partial discharge and the design of UHF sensor.

\section{THEORETICAL ANALYSIS OF TRANSMISSION} CHARACTERISTICS OF ELECTROMAGNETIC WAVE SIGNAL

When GIS appears defects, defect often inspire the partial discharge signals of extremely fast discharge time characteristics, the rapid rise of the partial discharge pulse generates nanosecond high frequency electromagnetic wave, at the same time TEM will inspire and generate a transverse electric wave (TE) and transverse magnetic (TM), and will cause resonance in the cavity body. Electromagnetic wave propagation in the coaxial structure damping is small, when UHF electromagnetic waves travel to the basin insulator that support of GIS inner conductor, UHF electromagnetic waves will leak out by metal flange pouring hole[9].

Metal pouring hole is rectangular waveguide structure, as shown in Fig .1. The long side is a, short side is b in the section of rectangular waveguide structure, the electric field lines is the direction of $y$ axis, magnetic induction 
line direction is the direction of $\mathrm{x}$ axis, the direction of $\mathrm{z}$ axis is the direction of propagation of electromagnetic wave in the rectangular waveguide structure.

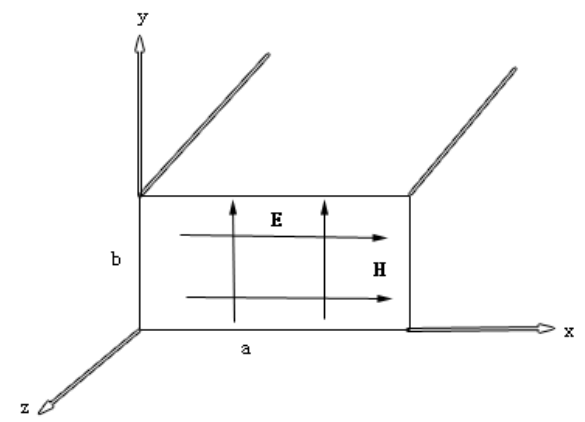

Figure 1. Rectangular cross section waveguide structure

Electromagnetic wave in rectangular waveguide structure should satisfy the Helmholtz equation and its boundary value, boundary condition,

$$
\left\{\begin{array}{l}
\nabla \times \stackrel{\rho}{H}=\varepsilon \partial \stackrel{\rho}{E} / \partial t \\
\nabla \times \stackrel{\rho}{\mathrm{E}}=-\mu \partial \stackrel{\rho}{H} / \partial t \\
\nabla \bullet \stackrel{\rho}{E}=0 \\
\nabla \bullet \stackrel{\rho}{H}=0
\end{array}\right.
$$

By (1),we can get wave equation,

$$
\left\{\begin{array}{l}
\nabla^{2} \stackrel{\rho}{E}+k^{2} \stackrel{\rho}{E}=0 \\
\nabla^{2} \stackrel{\rho}{B}+k^{2} \stackrel{\rho}{B}=0
\end{array}\right.
$$

Boundary value is,

$$
\left\{\begin{array}{l}
\rho \times\left(\stackrel{\rho}{E_{2}}-\stackrel{\rho}{E}_{1}\right)=0 \\
\rho \times(\rho-\rho \\
h \times\left(H_{2}-H_{1}\right)=\propto
\end{array}\right.
$$

The boundary condition of the electric field is,

$$
\left\{\begin{array}{l}
\text { 当 } \mathrm{x}=0, \mathrm{a} \text { 时, } E_{y}=E_{z}=\frac{\partial E_{x}}{\partial x}=0 \\
\text { 当 } y=0, b \text { 时, } \mathrm{E}_{x}=E_{z}=\frac{\partial E_{y}}{\partial y}=0
\end{array}\right.
$$

Rectangular waveguide structure is equivalent to a high pass filter. For TE, TM, their frequency must be greater than the cut-off frequency of each wave. Because of the TEM wave cannot build the static field, so TEM cannot spread in a rectangular wave structure. In a rectangular waveguide structure, the cut-off frequency of $\mathrm{TE}$ and TM is,

$$
f_{o c}=\frac{1}{2 \sqrt{\mu \varepsilon}} \sqrt{\left(\frac{m}{a}\right)^{2}+\left(\frac{n}{b}\right)^{2}}
$$

Among then, $\mu$ is magnetic permeability, $\varepsilon$ is dielectric constant, $\mathrm{a}$ and $\mathrm{b}$ are the length of sides in the cross section of rectangular waveguide structure. M, $\mathrm{n}=0,1,2,3 \ldots$ except that they are both zero.

\section{FDTD SIMULATION MODEL}

UHF signal propagation within the GIS cavity is reflected back and forth, also can produce resonance. According to the method introduced by literature[10], at the ends of the cavity with a matching cone structure can reduce the resonance, this paper will use this method to reduce the resonance.

GIS cavity size in simulation model is drawn based on the Shandong Tai Kai factory $126 \mathrm{kV}$ GIS. GIS inner and outer conductor is aluminum material, length $2000 \mathrm{~mm}$, diameter of outer conductor $244 \mathrm{~mm}$, diameter of inner conductor $60 \mathrm{~mm}$, cavity thickness $6 \mathrm{~mm}$, metal flange size $350 \times 350 \times 50 \mathrm{~mm}$, metal pouring hole size $50 \times 20 \mathrm{~mm}$, GIS cavity simulation model is shown in Fig. 2 . The key point of this paper don't include the attenuation of electromagnetic wave, simulation model design without adding insulator, only metal flange and a pouring hole are painted. The metal flange is consistent with the internal conductor and the external conductor materials, casting holes is filled by epoxy resin (dielectric constant 3.8), placing 5 probes in the metal flange casting hole surface, probe sensors position are shown in Fig. 3.

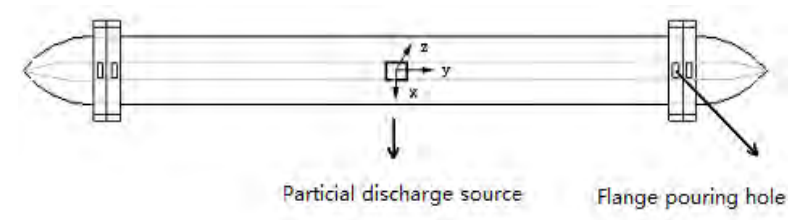

Figure 2. Simulation mode

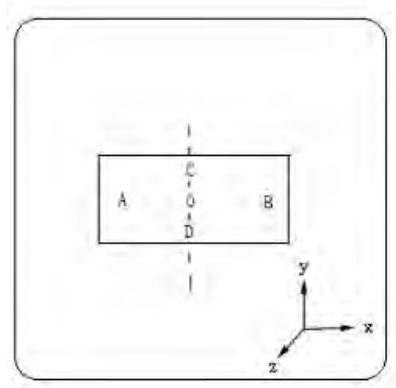

Figure 3. The position of probe sensor

\section{THE PARTIAL DISCHARGE SOURCE}

Partial discharge sources can be replaced by a Gauss pulse signal, the time domain form is,

$$
I(t)=I_{0} \exp \left(-\frac{4 \pi\left(\mathrm{t}-\mathrm{t}_{0}\right)^{2}}{\tau^{2}}\right)
$$

Among them, I0 is current pulse amplitude, $\tau$ is constant, $\tau$ determine the pulse width. When $\mathrm{t}=\mathrm{t} 0$, pulse reaches the maximum value. The gauss pulse used in the paper is shown in Fig. 4. 
Setting the highest simulation frequency is $5 \mathrm{GHz}$, light speed is $\mathrm{c}=3 \times 10^{8} \mathrm{~m} / \mathrm{s}$, maximum length Lmax in XFDTD cells is,

$$
L_{\max }=\frac{c}{10 \times f}=\frac{3 \times 10^{8}}{10 \times 5 \times 10^{9}}=6 \mathrm{~mm}
$$

FDTD is to replace MAXWELL curl equations for numerical calculation with a set of finite difference equations, with the growth of time step, numerical solution will be unstable [9]. According to the Courant stability condition, relation of time step and cell mesh size should satisfy the following conditions,

$$
c \Delta t \leq\left[\frac{1}{\Delta x^{2}}+\frac{1}{\Delta y^{2}}+\frac{1}{\Delta z^{2}}\right]^{-\frac{1}{2}}
$$

When $\quad \Delta \mathrm{x}=\Delta y=\Delta z \quad, \quad c \Delta t \leq \frac{\Delta x}{\sqrt{3}} \quad$,in general, $c \Delta t=\frac{\Delta x}{2}$

Setting the cell length is $3.6 \mathrm{~mm}$ in simulation, so the time step is,

$$
\Delta t=\frac{\Delta x}{2 c}=\frac{3.6 \times 10^{3}}{2 \times 3 \times 10^{-8}}=6 \times 10^{12} \mathrm{~s}
$$

The XFDTD signal source pulse width size is determined by the cutoff frequency of the rectangular waveguide structure and the time step. According to (5) and (9), the setting of the pulse width is 100 times the time step in the simulation.

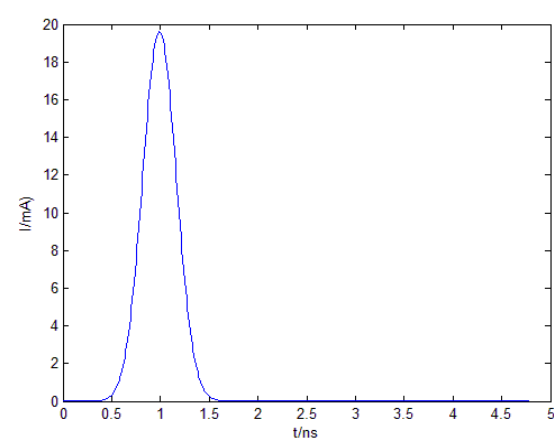

Figure 4. The pulsed wave of Gauss

\section{ANALYSIS OF SIMULATION RESULTS}

\section{A. Electric field analysis in point $O$}

Placed in the center metal flange casting hole surface, the electric field intensity in $\mathrm{x}, \mathrm{y}, \mathrm{z}$ direction measured by point sensor are shown in Fig. 5.
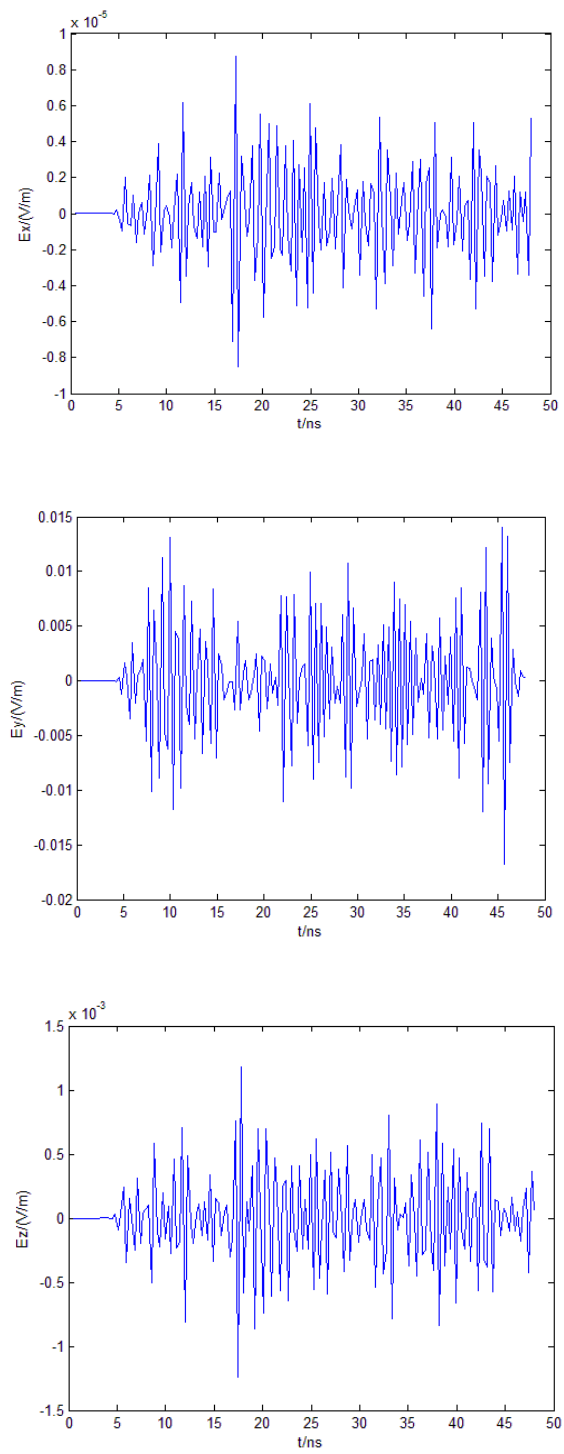

Figure 5. $x, y, z$ direction of the electric field intensity in point $\mathrm{O}$

The peak of electric field intensity in point $\mathrm{O}$ is shown in table 1 .

TABLE I. THE PEAK OF ELECTRIC FIELD INTENSITY IN POINT O

\begin{tabular}{cccc}
\hline E direction & Ex & Ey & Ez \\
\hline $\begin{array}{c}\text { Peak value } \\
\mathrm{mV} / \mathrm{m}\end{array}$ & 0.008 & 13.500 & 1.150 \\
\hline
\end{tabular}

From table 1, we see that Ey is the maximum electric field intensity modulus component in point $O$. The direction of y axis is GIS axial.

\section{B. Electric field analysis in point $A, B, C, D, O$}

The maximum electric field strength modulus value in point $\mathrm{A}, \mathrm{B}, \mathrm{C}, \mathrm{D}$ is in line with the $\mathrm{O}$ point, Ey direction is the maximum modulus value. Electric field intensity in Ey direction about points A, B, C, D are shown in Fig. 6. 

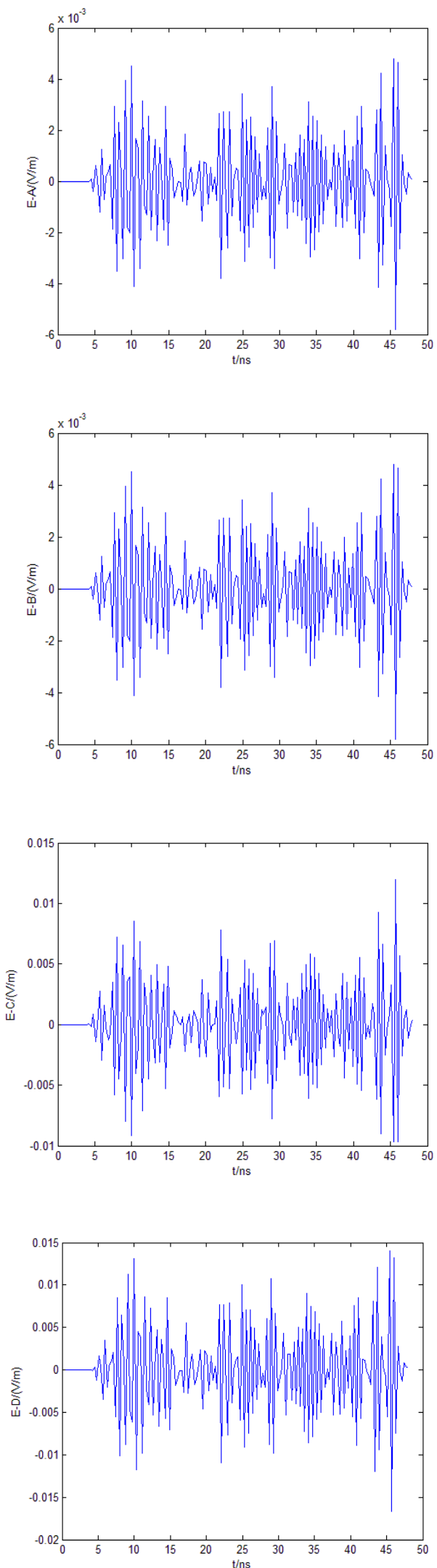

Figure 6. The $\mathrm{Y}$ axis direction of the electric field intensity in point A、B、C、D

The peak of electric field intensity in point A, B, C, D, $\mathrm{O}$ are shown in table 2. A、B、C、D、O

\begin{tabular}{cccccc}
\hline Point & A & B & C & D & O \\
\hline $\begin{array}{c}\text { Peak } \\
\text { value } \\
\mathrm{mV} / \mathrm{m}\end{array}$ & 4.80 & 4.80 & 12.00 & 14.50 & 13.50 \\
\hline
\end{tabular}

From table 2,we can see that electric field intensity is basically the same size in point C,D,O;A, B are the same electric field strength; the electric field strength modulus value in point $\mathrm{C} 、 \mathrm{D} 、 \mathrm{O}$ is about 3 times than $\mathrm{A}, \mathrm{B}$. The electric field intensity of metal flange pouring hole is decreasing along the long edge midline to both sides, both sides are similar. On the long side of midline location, field strength size is unchanged.

According to the above analysis, we should selection different position for different antenna types in the clockwise site layout of the external UHF sensor, the maximum receiving electromagnetic wave emitted from the pouring hole flange.

\section{CONCLUSION}

By using the finite difference time domain method, the author draws the following conclusions based on a large amount of simulation data and analysis:

(1)In the metal flange casting hole GIS can detect the partial discharge signal scattered from the hole and the maximum intensity component of the electric field direction is GIS axial.

(2)The module value of electric field intensity on the surface of the metal flange casting hole is about midline symmetrical, the center line (GIS axis) direction of the long side, the maximum electric field intensity component is similar.

\section{REFERENCES}

[1] Ni Guangzheng. Engineering electromagnetic field theory [M]. Beijing: higher education press, 2002.

[2] Li Xin, LIi Chengrong, Li Yasha, et al. Limited time domain method of differential points for the spread of the GIS partial discharge analysis [J]. Proceedings of the CSEE, 2005:150-155.

[3] PEARSON J S, HAMPTON B F, SELLAR A G. A continuous UHF monitor for gas-insulated substations [J]. IEEE Transactions on Dielectrics and Electrical Insulation, 1991,26(3):469-478.

[4] JUDD M D, FARISH O. Transfer function for UHF partial discharge signals in GIS [C]. High Voltage Engineering Symposium,London,UK, 1999,467:74-77.

[5] MEIJER S, SMIRT J J. UHF defect evaluation in gas insulated equipment [J]. IEEE Transaction on Dielectrics and Electrical Insulation,2005,12(2);285-296.

[6] Cheng Xu, Yan Tingli, Zhan Lumao, et al. Study on propagation properties of UHF signals of partial discharge in GIS [J]. High voltage apparatus,2014,05:30-35.

[7] Wang Kangning, Wang Tianzheng, Yan Jie etal. Typical insulation defects in GIS simulation and analysis of characteristics of partial discharge signals $[\mathrm{J}]$. Journal of electronic testing, 2013, 22:215-217.

[8] Li Lixue. Ultra high frequency detection of the envelope of partial discharge in GIS [D]. Shanghai Jiao Tong University,2009. 
[9] Liu Junhua, Wang Jiang, Qian Yong, et al. The electromagnetic wave propagation properties in GIS simulation [J]. High voltage technology, preceding 2007:139-142.
[10] Zhang Lei, Lin Qun, Ding Dengwei, et al. Simulation analysis of UHF GIS signal propagation characteristics of partial discharge $[\mathrm{J}]$. High voltage engineering, 2011,03:726-731. 